\title{
Die allgemeine progressive Paralyse bei Greisen.
}

\author{
Von \\ Prof. Dr. Juliano Moreira, \\ Direktor des Nationalen Irrenkrankenhauses in Rio de Janeiro, \\ und \\ Privatdozent Dr. Ulysses Vianna, \\ Primärarzt daselbst. \\ Mit 2 Textfiguren. \\ (Eingegangen am 12. Juni 1913.)
}

Brasilien ist seiner weiten Ausdehnung, der großen Mannigfaltigkeit der ethnischen Gruppen, welche es bewohnen, sowie auch der verschiedenen Bildung seiner Einwohner wegen noch immerfort eine vortreffliche Quelle wissenschaftlicher Studien.

Da nun die vergleichende Psychiatrie immer noch nach neuen Forschungen in ihrem Gebiete verlangt, kann man diese Vorteile Brasiliens auch für sie ausnützen.

In der Irrenanstalt zu Rio de Janeiro ist eine Reihe von Forschungen, welche sich zu Beiträgen für die verschiedenen Probleme der vergleichenden Psychiatrie eignen, angefangen worden. Die Resultate dieser Forschungen sollen in anderen Abhandlungen brasilianischer Ärzte in deutschen Zeitschriften veröffentlicht werden. Für heute werden wir es uns vorbehalten, uns mit 2 Fällen progressiver Paralyse zu beschäftigen, welche offenbar von Interesse sind, und zwar nicht nur weil es sich um progressive Paralyse bei alten Leuten, sondern auch noch nebenbei um Vollblutneger handelt.

Die senile progressive Paralyse ist noch besonderer Studien wert, weil sie wahrscheinlich weniger selten ist als man annimmt. Das Alter der Patienten hat hier wohl oft die Ärzte auf eine falsche Fährte geleitet und zu einer unrichtigen Diagnose veranlaßt. In den überfüllten Irrenanstalten (die ja in der Mehrzahl sind) kommt dieses am häutigsten vor.

Unsere 2 Fälle wurden z. B. längere Zeit falsch diagnostiziert, und selbst als einer von uns die richtige Diagnose feststellte, blieb dennoch bei einigen Kollegen Zweifel an der Richtigkeit, bis die diagnostischen Hilfsmittel (Untersuchung des Liquor spinalis, des Blutes) und die histopathologische Untersuchung die Diagnose absolut sicherstellte. 
Dieser Zweifel begründete sich hauptsächlich auf das hohe Alter der Patienten und ihrer Rasse.

Geben wir nun zunächst die kurze Geschichte unserer Patienten:

Fall I: J. Francisco, 99 Jahre alt, Brasilianer, Sohn eines afrikanischen Negers und einer afrikanischen Negerin (Fig. 1), wurde in die Pinelabteilung des Hospitals nacional de Alienados den 19. Juli 1910 vom S. Franciskus von Assisi Asyl (Haus für alte Leute) verlegt.

Anamnese: Kurze Zeit nach dem Eintritt in dieses Asyl, Sept. 1910, zeigte Pat. Verfolgungswahnideen. Er schrie des Nachts, daB man ihn bestehlen wollte, und da sich dieser Zustand immer mehr verschlimmerte, veranlaßte der Arzt des Asyls seine Überbringung in unsere Irrenanstalt.

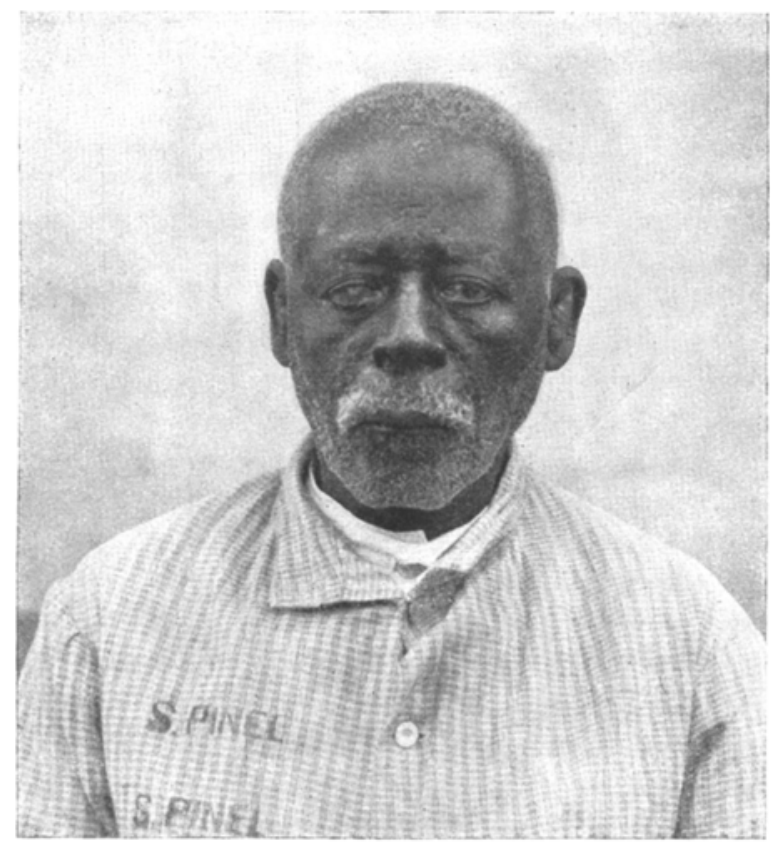

Fig. 1.

Nach anderer Auskunft erfuhren wir, daß Pat. niemals vorher in einer Irrenanstalt gewesen war und niemals gleiche psychische Erscheinungen gezeigt hatte, wie die, wegen welcher er in unsere Anstalt gebracht wurde, abgesehen von einer gewissen Reizbarkeit und einer schlechten Stimmung, welche wohl seinem hohen Alter zuzuschreiben waren. Pat. gibt selbst keine vertrauenswürdige Auskunft.

Objektiver Befund: Haare und Bart ganz weiß, 1,69 Größe, rechte obere Ohrenspitze exulceriert, großes linkes Othämatom. Asymmetrisches Gesicht, andere sogenannte Entartungszeichen und einige Rassenstigmata. Zähne im schlechten Zustande und unregelmäßig eingesetzt. Tiefer Gaumen und stark zitternde Zunge, nach links abweichend, fibrilläres Zittern der Lippenmuskulatur. Conjunctivitis beider Augen, Nebulae am linken Auge. Anisokorie; reflektorische Starre, Trägheit auf Konvergenz. 
Herz leicht hypertrophiert und diastolisches Herzgeräusch, schwacher Puls. Atmungsorgane ohne Besonderheit. Schmutzige Zunge. Koprostasis. Harnabgang. Wackeliger Gang in kleinen Schritten. Sensibilität kann nicht gut geprüft werden, jedenfalls wird nur auf tiefe Nadelstiche reagiert.

Patellarreflexe verringert, Abdominal-, Cremaster-, Plantarreflexe verschwunden. Babinski rechts. Pat. ist ruhig, gleichgültig. Blöder Gesichtsausdruck, spricht fortwährend leise. Ausgesprochene Dysarthrie. Wörter langgezogen und oft ausbleibend. Spricht die Paradigmata sehr schlecht nach. Fragen sehr schwer zu beantworten. Spricht dieselben oftmals nach, statt sie zu beantworten. Absurder Größenwahn, hält sich für reich, auf die Frage ob er ein Konto (ca. 1200 Mark) besäße, antwortet er, daß er 200 hätte und repetiert diesen Satz mehrere Male. Seine Wahnideen zeigen sich bei jeder Gelegenheit, sowie man mit ihm zu reden anfängt.

Auf die Bitte, zu flöten, antwortet er, daß er nur singen könne und fängt etwas über Joao Francisco (seinen eigenen Namen) der Gute usw. zu singen an und lächelt darauf. Unorientiert über Zeit und Umgebung. Keine Halluzinationen Aufmerksamkeit unnachhaltig. Ideenassoziationen fast nichtig.

Ausgesprochene Gedächtnisschwäche: Auf Nachfragen nach seinem Vorleben kommen verschiedene Antworten.

20. August: Nachts schmutzig. Kommt nachts mehrmals aus dem Bette heraus

Wassermannsche Reaktion (Originalmethode) in Spinalflüssigkeit pound läuft rastlos umher, leise vor sich hinmurmelnd.

7. Oktober Exitus letalis.

4 Stunden nach Feststellung des Todes erfolgte die Sektion. Schädeldach dick. Die Dura mater ist im Stirnteil mit dem Schädeldach verwachsen, straff gespannt und verdickt. Bei der Öffnung des Duralsackes entleert sich trübe, blutigseröse Flüssigkeit (ca. $100 \mathrm{ccm}$ ). In der rechten hinteren und mittleren Schädelgrube ist die harte Hirnhaut mit einer fibrinösen Neomembran belegt. Links war die Neomembran blutig infiltriert und klebte an der weichen Hirnhaut. Die weiche Hirnhaut war verdickt, mit der Rinde verwachsen, milchig, getrübt, besonders über Stirn und Parietalhirn. Gehirn verringert an Größe und Gewicht (1090 g). Das Gehirn zeigte Atrophie der Hirnwindungen im linken Hemispherium. Die Rindensubstanz verschmälert und braun-rosa. Die Marksubstanz derb und blutreich. Die Ventrikel etwas erweitert und mit deutlicher Ependymwucherung, Im Inneren der lateralen Ventrikel befand sich große Quantität von trüber Flüssigkeit. Die Plexus und die Tela Choroidea waren mit feinen Granulationen überdeckt. Die Dura mater spinalis dick und angeklebt an den Wirbelkanal. Die weiche Hirnhaut milchig. Die Gefäße angefüllt.

Außerdem fand sich bei der Sektion: Myokarditis, Sklerose der Valvula mitralis, Atherom der Aorta. Chronische Pleuritis, Oedema apicis, Hypostasis der Lungenbasis, Leber verkleinert. Perisplenitis, chronische Nephritis, linke Niere vergrößert, mit angeklebter Kapsel, der Unterpol abgerundet, zeigte beim Durchschneiden eine große Zone neugebildeten Gewebes, welches sich bis zur Rinde und Marksubstanz hin erstreckte.

Histologischer Befund: Pia verdickt. Große Infiltration von Plasmazellen und Lymphocyten. Die Plasmazellen in großer Mehrzahl, vielfach degeneriert. Die Ganglienzellen sind pigmentiert und zeigen daneben chronische und sklerotische Veränderungen. Die Alzhei merschen Fibrillenveränderung fehlt, auch die Redlich - Fischerschen Plaques. Die Gefäße sind vermehrt und infiltriert mit Plasmazellen. Zahlreiche Stäbchenzellen in den typischen Formen. Die Tangentialfasern verschwunden, die radiären und supraradiären Fasern vermindert. 
Fall II: A. A. de C., Brasilianer, 70 Jahre alt (Fig. 2), Sohn eines afrikanischen Negers und einer afrikanischen Negerin, Arbeiter, aufgenommen den 13. Okt. 1911.

Anamnese: Pat. selbst kann keine glaubhaften Angaben machen.

Status morbi: Individuum von 1,61 m Höhe, Körperbau mittelkräftig, Ernährungszustand mittelmäßig. Das Ansehen entspricht dem Alter. Asymmetrischer Gesichtsschädel. Große Ohren mit angewachsenen Läppchen. Prognatismus. Zähne verfault und schlecht sitzend. Rechter Oberarm in Abduktion, rechter Vorderarm gegen den Oberarm und die Finger gegen die Hand flektiert. Pupillen ungleich weit, reagieren nicht auf Lichteinfall. Herztöne ein wenig gedämpft, Puls gespannt. Beschmutzt sich. Zittern der Zunge. Zittern der oberen Glieder. Macht fast gar keine Bewegung, benutzt nur den linken Arm. Geht in kleinen Schritten, muß die Beine spreitzen, um das Gleichgewicht zu erhalten. Patellarreflexe rechts gesteigert und links abgeschwächt. Cremaster- und Plantarreflexe verschwunden. Abdominale Reflexe links anwesend und rechts abwesend. Sensibilität ein wenig abgestumpft.

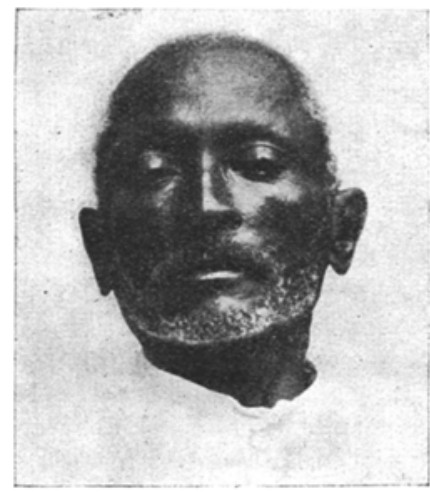

Fig. 2.

Psychischer Zustand: Blöder Gesichtsausdruck. Antwortet nur auf die Frage nach seinem Namen. Schwer von Begriff. Tut nicht, um was man ihn bittet, selbst den Mund macht er nur auf und zu, wenn man es ihm vormacht. Manchmal versucht er zu antworten, kann aber die Wörter nicht herausbekommen, artikuliert nur. Seinen Namen spricht er nur mit Mühe aus. Bei dieser Gelegenheit kann man ihm eine starke Dysarthrie anmerken. Unorientiert über Zeit und Ort. Sieht blöde auf seine Umgebung.

Wassermannsche Reaktion im Serum und der Spinalflüssigkeit positiv. Pleocytose. Am 22. Nov. 1911 starb Pat.

3 Stunden nach dem Tode erfolgte die Sektion.

Schädeldach dick. Die verdickte Dura mater ist im Stirnteil neben der Mittellinie und in der Nähe der Lambdanaht mit dem Schädeldach verwachsen. Die weiche Hirnhaut dick, milchig und mit der Rinde verwachsen. Das Gehirn an Größe und Gewicht verringert $(1280 \mathrm{~g})$. Die Hirnwindung verschmälert, die Hirnfurchen verbreitert. Seitenventrikel erweitert, deren Flüssigkeit klar und durchsichtig. Deutliche Ependymgranulationen in den Ventrikeln.

Die Basalgefäße sind im wesentlichen zart, voll von Blut. Die Häute des Rückenmarkes zeigen dieselben Veränderungen wie die des Gehirns.

Außerdem fanden sich Erweiterung, Hypertrophie und braune Degeneration des Herzens. Atherom der Aorta, verdickte Mitralklappen, ca. $30 \mathrm{ccm}$ Flüssigkeit im Herzbentel. Leichte Verwachsung der Lungenspitzen. Verkalkte Knötchen in beiden Lungenspitzen. Verdicktes Lungenfell an den Lungenspitzen. Chronische Bronchitis. Stark schwarz pigmentierte peribronchitische Drüsen. Leber vergröBert, Konsistenz verringert, auf dem Durchschnilt gelb rötlich. Milz verkleinert mit verdickter Kapsel. Chronische Nephritis.

Histologischer Befund: Dieselben Veränderungen wie im ersten Fall.

Diese histologischen Prüfungen sind im Laboratorium des Herrn Professor Alzheimer in Breslau gemacht. Wir haben die Verpflichtung, ihm vielfach zu danken! 
Wenn man die verschiedenen Statistiken über die Häufigkeit der allgemeinen Paralyse nachsieht, überzeugt man sich von der Seltenheit der Diagnose dieser Krankheitsfälle nach dem 6. Lebensjahrzehnt.

Noch kürzlich schrieb Hoche in seinem Artikel über Dementia paralytica im Handbuch der Psychiatrie, herausgegeben von Prof. Aschaffenburg: Fälle von progressiver Paralyse in den 70er Jahren sind nicht bekannt (S. 47).

Nach der Statistik von Junius und Arndt ist 67 das höchste Alter beim Ausbruch der Krankheit.

Siemerling fand von 284 Fällen allgemeiner Paralyse nur 6 Fälle $(2,1 \%)$ bei Patienten mit mehr als 60 Jahren.

Ziehen in seinem Lehrbuch der Psychiatrie sagt: Das Auftreten der Krankheit fällt am häufigsten in das 4. und 5. Lebensjahrzehnt (über $80 \%$ aller Fälle), am größten ist die Morbidität zwischen dem 35. und 45. Jahre (ca. 50\%). Im 3. Lebensjahrzehnt sowie im 6. ist sie bereits erheblich geringer.

Obersteiner zeigt uns in seiner Monographie über die allgemeine progressive Paralyse eine Statistik, bei der das höchste Alter bis 60 Jahre war. Was die späteren Fälle anbetrifft, sagt er folgendes: „Fälle von Paralyse nach dem 55. Jahre sind selten und suspekt, im Sinne einer anderen Erkrankung (Dementia senilis praecox)."

Nach der Statistik, die von Moreira und Penafiel 1906 veröffentlicht wurde, befanden sich unter 172 Fällen von Paralyse, welche im Hospital Nacional de Alienados zu Rio de Janeiro von 1889 bis 1904 aufgenommen worden waren, nur 4 Fälle zwischen dem 61 . bis 65. und 2 zwischen dem 66. und 70. Jahre. Alzheimer ist der erste gewesen, der progressive Paralyse im Senium mit klinischen Erscheinungen und histologischem Befund demonstriert hat.

Krae pelin in seinem Lehrbuch der Ps ychiatrie schreibt: ,Selbst im 7. Lebensjahrzehnt werden noch einzelne Fälle beobachtet" (S. 482).

Olivier suchte im Jahre 1906 alle bis dahin veröffentlichten Beobachtungen über senile progressive Paralyse kurz zusammenzufassen. Er verteilte dieselben in klinische Fälle ohne Nekropsie, klinische Fälle mit makroskopischer Untersuchung und klinische Fälle mit makroskopischer und mikroskopischer Untersuchung. Von der 1. Gruppe blieben 7 ohne Sektion. Wir glauben bei dem jetzigen Stand unserer Kenntnisse der pathologischen Anatomie der progressiven Paralyse diese Fälle für wissenschaftliche Beweise nicht benutzen zu können, zumą da auch neue wertvolle diagnostische Hilfsmittel (Untersuchung des Liquor spinalis, des Blutes) bei diesen 7 erwähnten Fällen nicht gemacht worden sind.

Fälle mit nur makroskopischer Untersuchung müssen als nicht beweiskräftig zurückstehen. Nur die besser beobachteten Fälle mit mikro- 
skopischer Untersuchung dürfen verwertet werden, um über die Frage, welche wir hier studieren, zu bindenden Schlüssen zu gelangen.

1906 veröffentlichte Olivier 5 bis dahin allein bekannte Fälle mit makroskopischer und mikroskopischer Untersuchung. Weiter finden sich in die Literatur folgende Fälle: 2 von Karpas, New York 1908, über eine Frau mit 65 Jahren und eine mit 70 Jahren, beide mit Obduktion und mikroskopischer Nachuntersuchung, einen von Takanori I keda bei einer 63 jährigen auch mit Obduktion (wegen des hohen Alters der Patientin nannte er die Krankheit Dementia paralytica presbiophrenica), einen von Trenel und Libert mit 70 Jahren ohne Obduktion, einen anderen derselben Autoren ohne Obduktion (zweifelhaft), ferner 2 Fälle von Trenel bei einer 60- und einer 67 jährigen Frau. Endlich sandte im Juni 1912 Dr. Sagel der Zeitschrift für die gesamte Neurologie und Psychiatrie noch einen kurzen Bericht über einen Fall von progressiver Paralyse bei einer 64jährigen Frau, von ihm pathologisch-anatomisch und serologisch untersucht.

In fast keinem von diesen veröffentlichten Fällen wurden Laboratoriumsuntersuchungen (Nonnes Vier Reaktion) usw. gemacht und noch weniger mikroskopische Untersuchungen im Sinne der Alzheimerschen und Niß1-Forschungen über allgemeine Paralyse und andere Geistesstörungen ausgeführt.

So erscheint wohl die Veröffentlichung unserer Fälle gerechtfertigt.

Außer dem Alter ist an unseren Patienten noch interessant, daß sie Kinder von echten afrikanischen Negern ohne Zeichen von Mischung mit anderen ethnischen Gruppen sind. Bei einer späteren Arbeit wird einer von uns (Moreira) noch eine größere Menge von Beobachtungen in bezug auf die Frage des Verhältnisses der ethnischen Zugehörigkeit zu den Geisteskrankheiten in Brasilien veröffentlichen. Für heute wiederholen wir nur das, was einer von uns (Moreira) allein oder in Zusammenarbeit mit Peixoto oder Panafiel schon in früheren Arbeiten berichtete: Our statistics show that there is no race immunity from the various forms of insanity. Some years ago it was thought that the negro was exempt from the disease under discussion (dementia paralytica) ... We ourselves have observed the disease in members of all the ethnical groups inhabiting Brasil, with the exeption of pure aborigine and the directly imported African negro. The disease frequently assumed the apathetic form, and there was nothing in the symptommatology peculiar to the various groups. Such differences as did exist were individual and due to the degree of civilisation and education attained by the patient. (Moreira and Penafiel - Dementia paralytica in Brasil. J. of M. S.)

Im Amsterdamer Kongreß bezeugte Moreira: D'ailleurs nous a vons la conviction que la rareté de la démence paralytique dans les stati- 
stiques des principaux centres du Bresil est plus grande que la réalité. Cela tient pour la plupart a des erreurs de diagnostic. Beaucoup de médecins et des plus instruits, meconnaissent la paralysie générale là où un alieniste pracicien n'hésite pas à l'affimer et ne consentent à l'admettre que lorsque le syndrome est complet."

Heute, nachdem die Wassermannsche Reaktion und andere Laboratoriumsmethoden zur Verfeinerung der Diagnose im Gebrauch sind, wurde unsere Utberzeugung, daß die Paralyse häufiger bei uns vorkommt als man annimmt, noch vergrößert. Was die Frauen und Farbigen anbetrifft, haben wir dafür sehr wertvolle Beweise.

Wie wir schon oben erwähnt haben, sind die Fälle, über welche wir hier berichteten, zunächst als senile Demenz angesehen worden. Auch haben wir in Brasilien beobachtet, daß im allgemeinen bei Frauen und Farbigen von geringerer Bildung die Größenwahnideen sehr schnell im Verlaufe der progressiven Paralyse verschwinden, und es ist wohl anzunehmen, daß das Verschwinden dieses auffallenden Symptoms früher sehr oft die Ärzte auf falsche Bahnen geleitet hat.

Sagt ja auch Näcke: ,Trotz der verfeinerten Diagnose kommen immer noch Fälle verkannter Paralyse vor."

Was nun die Neger im allgemeinen anbetrifft, so bezeugen unsere Fälle noch einmal, daß es keine Rassenimmunität gibt gegen die progressive Paralyse, sondern daß die Zivilisation einen solchen Einfluß auf die Neger ausgeübt hat, daß ihre Nachkommen auch die Krankheiten, welche man im unzivilisierten Teil Afrikas nahezu nicht kannte, bekommen.

Noch eine letzte Frage muß bei unseren 2 Fällen erörtert werden: Sind es alte Fälle, stationäre Paralyse, Fälle von protrahiertem Verlauf oder im Gegenteil sind es Fälle bei Greisen, welche sich im späteren Alter mit Syphilis infizierten und paralytisch wurden. Wir haben keine sicheren Anhaltspunkte, um die Frage zu lösen, weil wir keine sicheren Angaben über die Vorgeschichte haben.

\section{Literaturverzeichnis.}

Alzheimer, Histologische Studien zur Differenzialdiagnose der progressiven Paralyse. Nissls Arbeiten. 1904.

Junius und Arndt, Beiträge zur Statistik, Ätiologie, Symptomatologie und pathologischen Anatomie der progressiven Paralyse. Arch. f. Psych. 44, 249. 1908.

Siemerling, Geistes- und Nervenkrankheiten des Greisenalters. Herausg. v. Schwalbe. 1909.

Ziehen, Psychiatrie, 4. Aufl. 1911.

Kraepelin, Psychiatrie 2, 1. Teil. 1910.

Obersteiner, Die progressive allgem. Paralyse, 2. Aufl. 1908.

Moreire und Penafiel, A contribution to the Study of Dementia paralytica in Brazil. Journ. of Mental Science. London 1907.

z. f. d. g. Neur. u. Psych. O. XVIIr. 
194 J. Moreira u. U. Vianna: Die allgemeine progressive Paralyse bei Greisen.

Moreira und Peixoto, Les maladies mentales au Brésil. I. Congrès International de Psychiatrie. Amsterdam 1907.

Olivier, De la paralysie générale sénile. Revue de Psych. 1906.

Karpas, General paralysis in the senile period etc. N.-Y. Med. Journ., 25th Jan. 1908 - apud Campbell in Review of Neurology and Psychiatriy. 1908.

Takanori Ikeda, Ein Fall von Pachymeningitis hamorrhagica interna bei der progressiven Paralyse. Shinkeigaku Zasshi (Neurologia) 8, Nr. 3-4. apud. d. deutsche Ausgabe 3, Tokio 1911, St. 72 und Jahresbericht der Neurologie und Psychiatrie, 1909.

TreneI und Libert, Paralysie generale senile et presbyophrenie. Bulletin de la Société Clinique de Med. Mental. N. 7. 1910.

Trene1, Paralyse générale sénile et conjugale. Bulletin de la Soc. Clinique de Med. Mentale Nr. 7. 1911.

Sagel, UUber einen senilen Fall von progressiver Paralyse. Zeitschr. f. d. ges. Neur. u. Psych. St. 651. Berlin 1912.

Näcke, Über atypische Paralysen. Allg. Zeitschr. f. Psych. 2. Heft. 1910. 